\title{
Ontogenetic Changes of Lipofuscin-Like Pigments in the Rat Heart
}

\section{J. WILHELM ${ }^{1,2}$, I. OŠŤÁDALOVÁ ${ }^{1,2}$}

${ }^{1}$ Centre for Cardiovascular Research, Prague, Czech Republic, ${ }^{2}$ Institute of Physiology, Academy of Sciences of the Czech Republic, Prague, Czech Republic

Received April 2, 2012

Accepted May 15, 2012

\section{Summary}

Increased generation of reactive oxygen species results in the formation of fluorescent end-products of lipid peroxidation lipofuscin-like pigments (LFP). LFP increased up to six-fold from the fetal value in the rat heart immediately after birth. In the experimental design of this study the fetuses were sampled 1 day before birth, and then the samples were collected on postnatal days $1,4,7,10,15,30$, and 60 . Males and females were compared on day 30 and 60 when the difference between right and left ventricle was studied as well. Four LFP fluorophores were analyzed: F355/440, F310/470, F350/450, F315/450 (excitation/emission, $\mathrm{nm}$ ). All fluorophores decreased on day 4 relative to day 1 , subsequent transient increases ended in a significant decrease on day 60 . However, the LFP levels on day 60 are still about threefold higher than those in fetuses. Differences between male and female hearts were observed on day 30 . The corresponding male ventricles contained by one third higher concentration of LFP than the female counterparts. The increase in LFP concentration in male ventricles on day 30 was only transient, no difference between corresponding male and female ventricles was found on day 60 . The most distinguished feature in the male heart was a sharp LFP decrease in the right ventricle on day 60 .

\section{Key words}

Rat heart • Ontogenetic development • Lipofuscin-like pigments (LFP) • Reactive oxygen species (ROS) • Gender difference • Right /left ventricle ratio

\section{Corresponding author}

J. Wilhelm, Department of Membrane Receptor Biochemistry, Institute of Physiology, Academy of Sciences of the Czech Republic, Vídeňská 1083, 14220 Prague 4, Czech Republic. E-mail: wilhelm@biomed.cas.cz

\section{Introduction}

Immediately after birth, body oxygen concentration undergoes dramatic changes that, from the quantitative point of view, are comparable to transition of experimental animals from normoxia to hyperoxia. Hyperoxic stress in adults was documented as a cause of heart oxidative damage (Nohl et al. 1981), and accordingly, an increase in reactive oxygen species (ROS) in the heart after birth is therefore to be expected. We have found the changes corresponding to increased ROS in the rat heart shortly after birth (Ošt'ádalová et al. 2007, 2010). The end-products of lipid peroxidation, the so called lipofuscin-like pigments (LFP), are widely used to assess the extent of oxidative damage (Jamieson 1991, Shimasaki 1994, Vasankari et al. 1995, Wilhelm and Herget 1999). These fluorescent lipophilic substances were originally named on the basis of the similarity of their fluorescence properties with those of lipofuscin the pigment of old age (Chio et al. 1969a). However, later studies showed that they are not directly related to lipofuscin formation, but they are rather the result of free radical-initiated oxidative damage to membrane lipids (Armstrong et al. 1992).

The compounds originate from reactions between malonaldehyde (MDA), the lipid peroxidation product, and amino acids (Chio et al. 1969b). Their emission maximum is at $450-470 \mathrm{~nm}$ after the excitation by ultraviolet light. Two excitation maxima were found at 260-280 $\mathrm{nm}$ and in the region between 350-390 $\mathrm{nm}$ (Trombly and Tappel 1975). Infrared and mass spectral analysis proved the presence of aminoiminopropene $(-\mathrm{N}=\mathrm{CH}-\mathrm{CH}=\mathrm{CH}-\mathrm{NH}-)$ structure (Tappel 1975). Reaction of MDA with other primary amines leads to the formation of another group of fluorescent compounds, 
1,4-dihydropyridine-3,5-dicarbaldehydes (Kikugawa et al. 1981). These compounds exhibit fluorescence emission maxima at $435-465 \mathrm{~nm}$ when excited at 365-405 nm. In addition, MDA can produce fluorescent compounds during polymerization (Gutteridge et al. 1977). Analogical compounds to those produced by the reactions of MDA are formed from other aldehydes originating from lipid peroxidation, such as 4-hydroxynonenal HNE (Esterbauer et al. 1986). Additionally, numerous fluorescent products have been obtained following reactions between primary amines and lipid peroxidation products other than MDA and HNE (Jamieson 1991).

The data on the ontogenetic development of LFP formation in the rat heart are very scarce. In the previous studies only a couple of LFP fluorophores in a several age periods were studied (Csallany et al. 1986, Ošt'ádalová et al. 2007, 2010). Therefore we set up the present study to describe deeply the time course of oxidative change in the rat heart starting from prenatal up to adult postnatal ontogeny in great detail, aiming also to the sexual differences and the difference between particular compartments of the heart. A complex picture of LFP changes during early ontogenetic development is revealed.

\section{Methods}

\section{Animal model}

The study was conducted in accordance with the Guide for the Care and Use of Laboratory Animals published by the US National Institutes of Health (NIH publication No. 85-23, revised 1996).

Eight pregnant female Wistar rats were used throughout the experiments. They had free access to water and standard laboratory diet. The offsprings (a total of 80 neonates) of both sexes were investigated 1 day before birth, and then on days $1,4,7,10,15,30$, and 60 of postnatal life. All animals were weighed and killed by cervical dislocation. Each experimental group of neonates $(n=8)$ was composed of at least three different nests.

After killing the animals the chest was quickly opened, the heart was rapidly excised, the atria were removed and the ventricles were weighed, frozen and used for analysis. In groups sampled on day 30 and day 60 the animals were divided according to sex and the right ventricle and the left ventricle together with septum were separated.

\section{Fluorescence analysis}

The technique described by Goldstein and McDonagh (1976), as modified by Wilhelm and Herget (1999), was used for the analysis of LFP in heart homogenates. Approximately $30 \mathrm{mg}$ of frozen heart sample was weighed, chopped to fine pieces, and transferred into a glass-stoppered test tube containing $6 \mathrm{ml}$ of chloroform-methanol mixture $(2: 1, \mathrm{v} / \mathrm{v})$. After $1 \mathrm{~h}$ extraction on a motor-driven shaker, $2 \mathrm{ml}$ of double distilled water was added, the sample was agitated and the ensuing mixture was centrifuged (400 g, $10 \mathrm{~min}$ ). After centrifugation, the lower chloroform phase was separated and used for measurements.

The quantitative estimation of LFP was based on excitation and emission maxima found in tridimensional spectral arrays. We identified four major fluorophores F365/440, F310/470, F350/450, and F315/450 (excitation/emission, $\mathrm{nm}$ ). The fluorometer was calibrated with the standard No. 5 of the instrument manufacturer and the LFP concentration was expressed in relative fluorescence units (rfu) per mg tissue wet weight.

\section{Statistical analysis}

Each observation was obtained from at least eight heart preparations in the group. Differences among the groups were evaluated using two- and one-way analysis of variance. For pairwise mean comparisons, the Student-Newman-Keuls multiple range test was applied. All the used programs belong to BMDP Statistical Software, University of California. Differences were considered as statistically significant when $\mathrm{p}<0.05$.

\section{Results}

In comparison with the fetal level, all the fluorophores increased 2.5-6 fold on postnatal day 1. In order to enable a direct comparison of the time course of all these fluorophores, we expressed their concentrations as a percentage of the respective fetal level that was set as $100 \%$. Then the significance of concentration changes during further development was related to the level on day 1 . The summary of quantitative changes of individual fluorophores in the course of development is shown in Figure 1. Our preliminary histochemical study has shown no differences between left and right ventricle or differences between males and females up to day 15 post partum, we therefore used whole male hearts for the assay. 


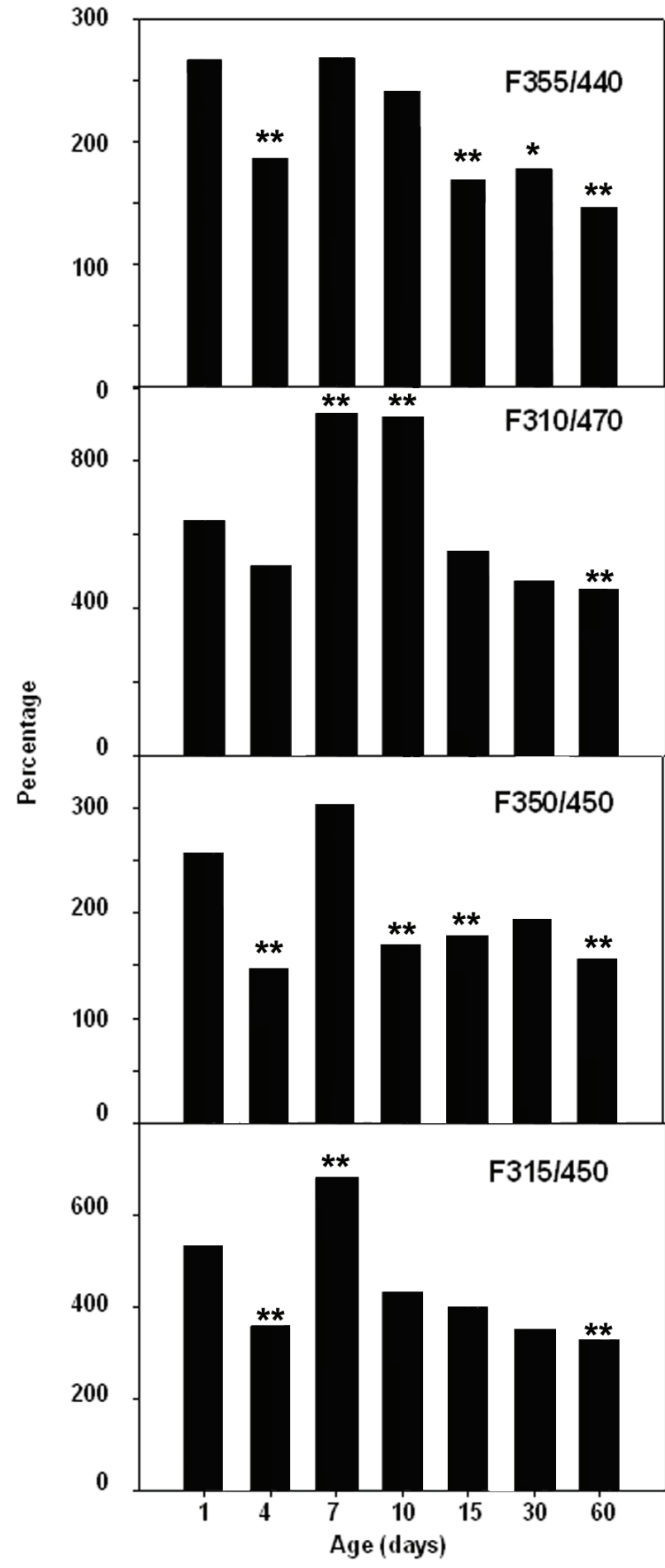

Fig. 1. Time-course of individual fluorophores during heart development. The percentage is related to the fetal value, taken as $100 \%$. Statistical significance evaluates the change in comparison with the value of day $1: * p<0.05, * * p<0.01$.

It is apparent from the time course of the concentrations of individual fluorophores, that they posses specific features and thus represent different chemical species. However, some features are common for more than one fluorophore. First, it is the decrease on the day 4, which is significant for all fluorophores except
F310/470. Second, all fluorophores are decreased on day 60 in relation to day 1 . Otherwise, there was an increase on day 7 in F310/470 and F315/450. The behavior of F310/470 and F350/450 fluorophores markedly differed from each other as well as from other fluorophores on day 10 when F310/470 was significantly increased and F350/450 was significantly decreased, while other fluorophores were not changed.

First differences between right and left ventricles and between male and female hearts were observed from day 30 on. Therefore we investigated individual fluorofores with this regard on days 30 and 60. For the statistical evaluation paired comparisons were used. In order to detect even the small changes we expressed the LFP concentration in absolute values (rfu/mg tissue wet weight).

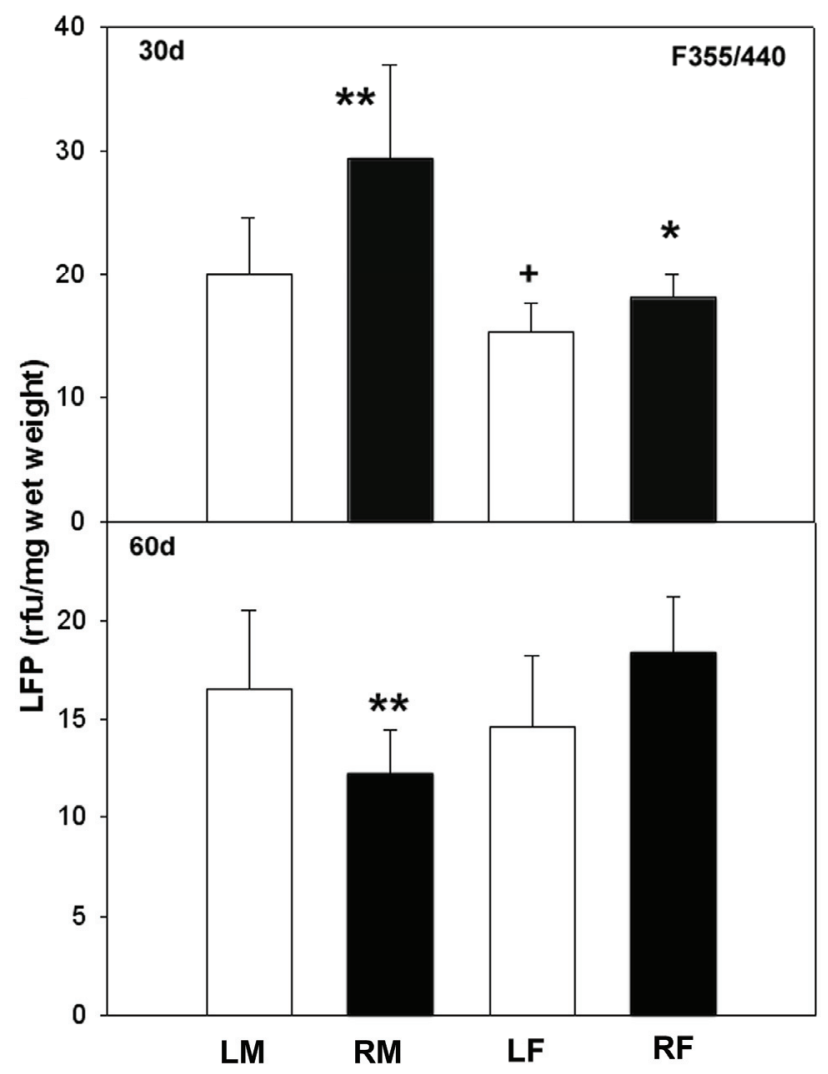

Fig. 2. Concentration of fluorophore F355/440. Upper panel day 30, lower panel day 60. Abbreviations: LM - left male ventricle with septum, RM - right male ventricle, LF -left female ventricle with septum, RF - right female ventricle. Statistical significance between heart compartments: $* \mathrm{p}<0.05, * * \mathrm{p}<0.01$, statistical significance between male and female: ${ }^{+} p<0.05$.

Figure 2 shows the changes of F355/440. On day 30 , it increased in the right ventricle relative to the left ventricle both in males and females. The concentration in females was significantly lower than in males. On day 60 F355/440 significantly decreased in male right ventricle, 


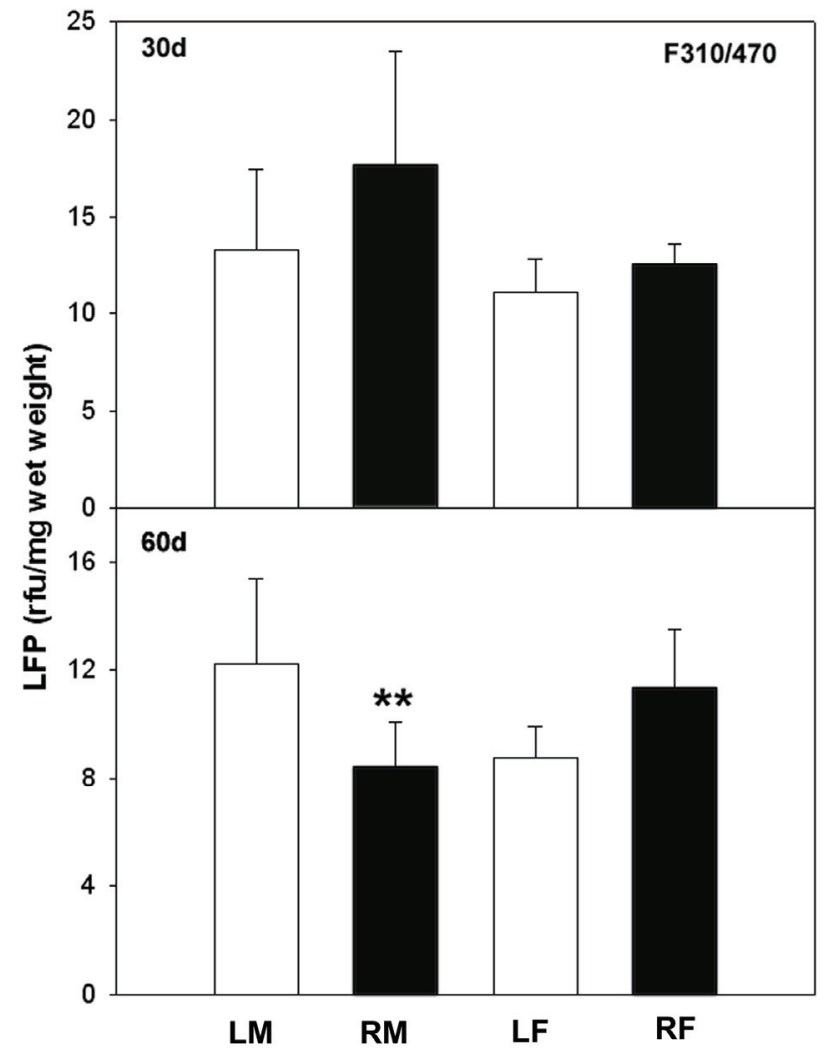

Fig. 3. Concentration of fluorophore F310/470. For the description see Figure 2.

which was opposite to day 30 . In female heart no changes were observed and there was no difference between males and females.

The changes of F310/470 are shown in Figure 3. On day 30 , there were no changes between ventricles or sexes. The tentency was similar as with F355/440 but due to higher dispersion of the data the difference was not significant. The only change on day 60 was a decreased concentration in male right ventricle, no difference was observed between male and female heart on day 60 .

The situation of F350/450 is illustrated in Figure 4. On day 30 , there were no changes in male heart. In females the concentration was increased in the right ventricle as compared to the left ventricle, and both concentrations were lower in females than in males. The overall pattern was similar to F355/440. The only change on day 60 was again a decreased concentration in male right ventricle, while no change was observed between male and female heart.

The changes of F315/450 are given in Figure 5. There is a single significant decrease in right male ventricle on day 60 . No significant changes were observed in all other comparisons. Anyhow, the pattern resembles all other fluorophores.

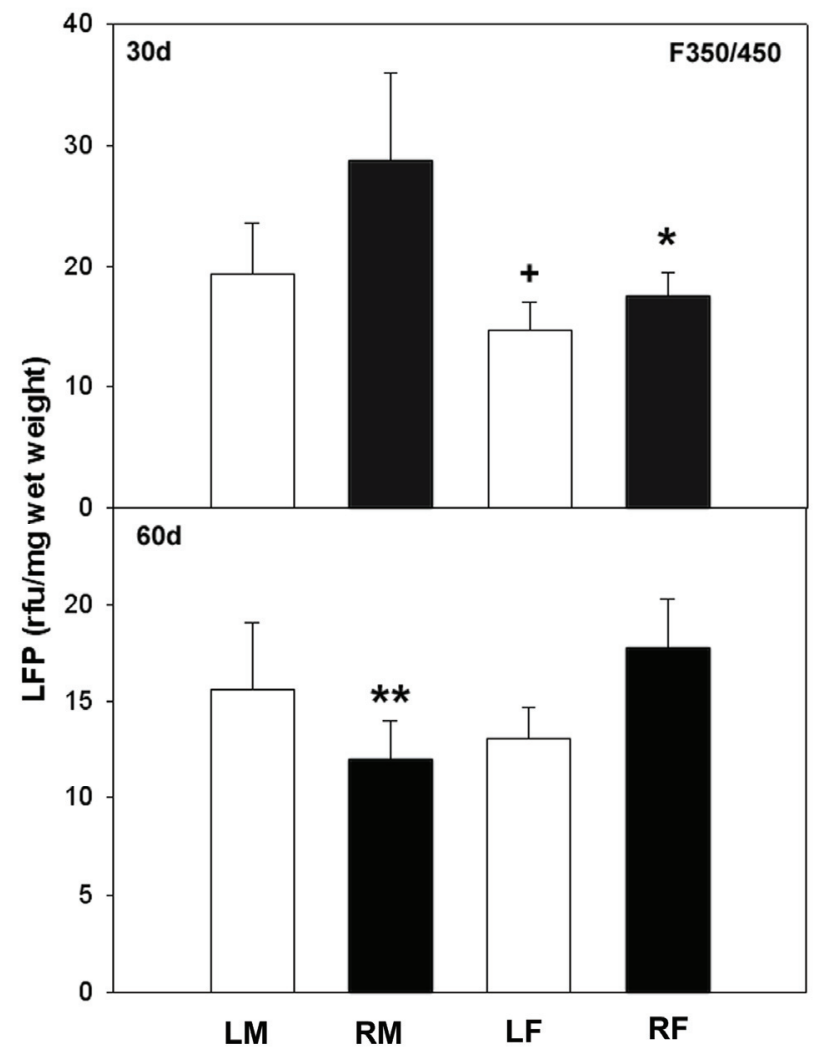

Fig. 4. Concentration of fluorophore F350/450. For the description see Figure 2.

A characteristic feature appears in the right/left ventricle ratio of LFP concentration in males and females on day 30 and 60. As shown in Figure 6, there is no significant gender difference in the ratio in all fluorophores on day 30 - production of LFP prevails in right ventricles. On day 60 the same situation persists in female hearts, whereas in male hearts all fluorophores significantly decrease and LFP concentration prevails in the left ventricle and septum.

\section{Discussion}

The changes in LFP concentration during heart development are multiple and complex, but they show few common characteristics. On the first postnatal day there is a multifold increase in LFP concentration in relation to the prenatal values. It thus appears that transition of the organism to air breathing is accompanied with oxidative stress. After delivery, tissue $\mathrm{P}_{\mathrm{O} 2}$ increases more than threefold (from 47 to $160 \mathrm{~mm} \mathrm{Hg}$ ) and arterial $\mathrm{O}_{2}$ saturation increases from 18 to $97 \%$. These conditions create an oxidative stress in the tissue adapted to the fetal oxygen levels. This transition is accompanied by a change in nutrition because transplacental supply of 


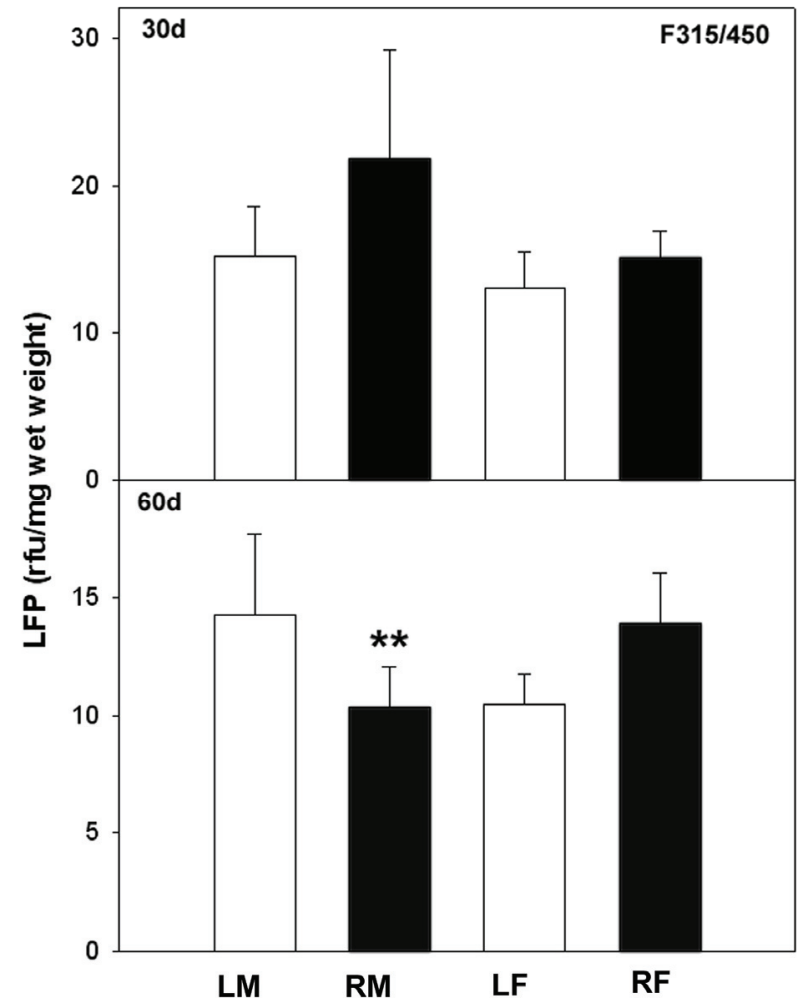

Fig. 5. Concentration of fluorophore F315/450. For the description see Figure 2.

nutrients is interrupted and mother milk nutrition is not yet fully developed (Kuma et al. 2004).

Oxidative stress develops as an imbalance between the production of reactive oxygen species and the antioxidant defense. However, the simple assumption that ROS are always detrimental to the organism and that high levels of antioxidants must be beneficial due to their scavenging properties is no longer acceptable (Upham and Trosko 2009). Thus ROS are now not only a byproduct of metabolism, they are utilized as a critical regulator of multiple intracellular signaling cascades. Therefore, the increase in ROS, followed by the increase of LFP on day 1 is probably very important for the next cardiac development.

Most of the LFP fluorophores decrease on the $4^{\text {th }}$ day after birth. It is not easy to explain these changes, however, day 4 appears to be critical for the postnatal cardiac development in the rat and it is a very important turning point in the rat myocardial remodelling. At this age cellular proliferation (hyperplasia) in rat heart decreases, predominantly hypertrophic growth occurs ( $\mathrm{Li}$ et al. 1996), and the value of heart/body weight ratio is the highest (Ošt'ádal et al. 1967, Ošt’ádalová et al. 1993).

Some of the fluorophores show a transient increase between day 7-10. This period is characterized

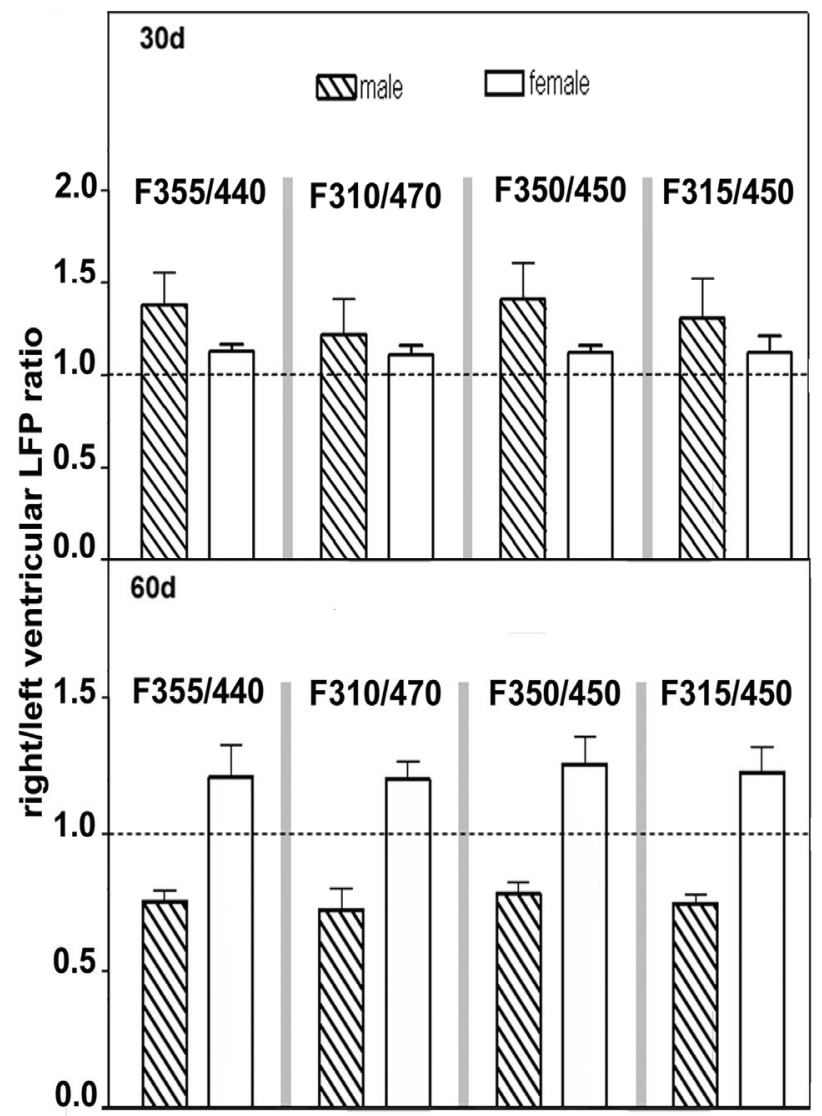

Fig. 6. Right/left ventricle (plus septum) ratio of LFP in males and females. Upper panel day 30, lower panel day 60 .

by intensive body growth with considerable daily body weight increments (Babický et al. 1972). High metabolic rate is generally accompanied by the increase in heart metabolism and increased ROS production which results in higher LFP levels.

On day 60 all fluorophores in male heart are significantly decreased to approximately one half in comparison with their levels on day 1. Sixty-day-old animals are sexually mature and we might expect that young healthy animals do not possess signs of oxidative damage. However, the LFP levels are still about threefold higher than those in fetuses. It thus appears that intensive aerobic metabolism of the heart is accompanied with certain basal level of ROS production.

We found substantial changes between male and female hearts on day 30. By absolute amount, the corresponding male ventricles contained by one third higher concentration of LFP than the female counterparts. This difference was in some fluorophores statistically significant (F350/450, F355/440), while in the others the significance was blurred by great data scatter. The measurement of LFP in the heart is generally accompanied by great data scatter. Besides the technical 
reasons ensuing from processing minute amounts of tissue, the cause can be also biological. In the recent study Wilhelm et al. (2011) documented a higher production of free radicals in the vicinity of blood vessels. Thus the uneven vascularity of the samples can add to the dispersion of measured LFP concentration. Anyhow, the increase in LFP concentration in male ventricles on day 30 was only transient, as no difference between corresponding male and female ventricles was found on day 60 .

Further characteristic feature of day 30 is the increased concentration of LFP in the right ventricle over the left ventricle with septum. This was observed in both males and females. It indicates specific processes comprising free radicals that are running in the right ventricle. However, on day 60 the situation changes dramatically. LFP concentration in the right male ventricle decreases to one half of that on day 30 , while the concentration in the left male ventricle and in both female ventricles is unchanged. If we express the right/left LFP ventricular ratio (Fig. 6), a higher LFP concentration is in the right ventricles for both males and females on day 30, however, on day 60 a higher production of LFP remains in right ventricles in females only, whereas in males a significantly higher LFP concentration was observed in the other compartments of the heart. It is not easy to explain the results. At the age of 60 days the rats are fully sexually maturated. In males both body size and left ventricle weight considerably increase. These changes might be probably accompanied by a higher metabolic activity and ROS production. However, this process can be more complex and needs future studies.

\section{Conflict of Interest}

There is no conflict of interest.

\section{Acknowledgements}

This study was supported by grant of GAČR P303/11/0298.

\section{References}

ARMSTRONG D, WILHELM J, ŠMÍD F, ELLEDER M: Chromatography and spectrofluorometry of brain fluorophores in neuronal ceroid lipofuscinosis (NCL). Mech Ageing Dev 64: 293-302, 1992.

BABICKÝ A, PǍ̌ÍZEK J, OŠŤÁDALOVÁ I, KOLÁŘ J: Initial solid food intake andgrowth of young rats in nests of different size. Physiol Bohemoslov 22: 557-566, 1972.

CHIO KS, REISS V, FLETCHER B, TAPPEL AL: Peroxidation of subcellular organelles: Formation of lipofuscin-like pigments. Science 166: 1535-1536, 1969a.

CHIO KS, TAPPEL AL: Inactivation of ribonuclease and other enzymes by peroxidizing lipids and by malonaldehyde. Biochemistry 8: 2827-2832, 1969 b.

CSALLANY AS, AYAZ KL, MENKEN BZ: Organic solvent soluble lipofuscin pigments and glutathione peroxidase in weanling and old rats. Int J Vitam Nutr Res 56: 143-147, 1986.

ESTERBAUER K, KOLLER E, SLEE RG, KOSTER JF: Possible involvement of the lipid-peroxidation product 4-hydroxynonenal in the formation of fluorescent chromolipids. Biochem J 239: 405-409, 1986.

GOLDSTEIN BD, MCDONAGH EM: Spectrofluorescent detection of in vivo red cell lipid peroxidation in patients treated with diaminodiphenylsulfone. J Clin Invest 57: 1302-1307, 1976.

GUTTERIDGE JMC, HEYS AD, LUNEC J: Fluorescent malondialdehyde polymers from hydrolyzed 1,1,3,3tetramethoxy propane. Anal Chim Acta 94: 209-211, 1977.

JAMIESON DD: Lipid peroxidation in brain and lungs from mice exposed to hyperoxia. Biochem Pharmacol 41: 749$756,1991$.

KIKUGAWA K, MACHIDA Y, KIDA M, KURECHI T: Studies on peroxidized lipid. III. Fluorescent pigments derived from the reaction of malonaldehyde and amino acids. Chem Pharm Bull 29: 3003-3011, 1981.

KUMA A, HATANO M, MATSUI M, YAMAMOTO A, NAKAYA H, YOSHIMORI T, OHSUMI Y, TOKUHISA T, MIZUSHIMA N: The role of autophagy during the early neonatal starvation period. Nature 432: 1032-1036, 2004.

LI F, WANG X, CAPASSO JM, GERDES AM: Rapid transition of cardiac myocytes from hyperplasia to hypertrophy during postnatal development. J Mol Cell Cardiol 28: 1737-1746, 1996. 
NOHL H, HEGNER D, SUMMER K-H: The mechanism of toxic action of hyperbaric oxygenation on the mitochondria of rat heart cells. Biochem Pharmacol 30: 1753-1757, 1981.

OŠŤÁDAL B, WACHTLOVÁ M, BÍLÝ J, RAKUŠAN K, POUPA O: Weight of the heart in the rat before and after birth. Physiol Bohemoslov 16: 111-115, 1967.

OŠŤÁDALOVÁ I, KOLÁŘ F, OŠŤÁDAL B, ROHLÍČEK V, ROHLIIČEK J, PROCHÁZKA J: Early postnatal development of contractile performance and responsiveness to $\mathrm{Ca}^{2+}$, verapamil and ryanodine in the isolated rat heart. J Mol Cell Cardiol 25: 733-740, 1993.

OŠŤÁDALOVÁ I, VOBECKÝ M, CHVOJKOVÁ Z, MIKOVÁ D, HAMPL V, WILHELM J, OŠŤÁDAL B: Selenium protects the immature heart against ischemia/reperfusion injury. Mol Cell Biochem 300: 259-267, 2007.

OŠŤÁDALOVÁ I, CHARVÁTOVÁ Z, WILHELM J: Lipofuscin-like pigments in the rat heart during early postnatal development: effect of selenium supplementation. Physiol Res 59: 881-886, 2010.

SHIMASAKI H: Assay of fluorescent lipid peroxidation products. Methods Enzymol 233: 338-346, 1994.

TAPPEL AL: Lipid peroxidation and fluorescent molecular damage to membranes. In: Pathobiology of Cell Membranes. Vol I. BF TRUMP, AU ARSTILA (eds), Academic Press, New York, 1975, pp 145-170.

TROMBLY R, TAPPEL A: Fractionation and analysis of fluorescent products of lipid peroxidation. Lipids 10: 441$447,1975$.

UPHAM BL, TROSKO JE: Oxidative-dependent integration of signal transduction with intercellular gap junctional communication in the control of gene expression. Antiox Redox Signal 11: 297-307, 2009.

VASANKARI T, KUJALU U, HEINONEN O, KAPANEN J, AHOTUPA M: Measurement of serum lipid peroxidation during exercise using three different methods: diene conjugation, thiobarbituric acid reactive material and fluorescent chromolipids. Clin Chim Acta 234: 63-69, 1995.

WILHELM J, HERGET J: Hypoxia induces free radical damage to rat erythrocytes and spleen: analysis of the fluorescent end-products of lipid peroxidation. Int J Biochem Cell Biol 31: 671-681, 1999.

WILHELM J, OŠŤÁDALOVÁ I, VYTÁŠEK R, VAJNER L: Generation of hydrogen peroxide in the developing rat heart: the role of elastin metabolism. Mol Cell Biochem 358: 215-220, 2011. 